FORMATION Formation emploi

Revue française de sciences sociales

147 | Juillet-Septembre

Italie : réformes politiques et permanences sociétales

des relations formation-emploi

\title{
Le marché du travail des diplômés universitaires en Italie : entre perspectives de reprise et difficultés structurelles
}

Italy's graduate labour market : possible economic recovery and structural

challenges

Der Arbeitsmarkt für Hochschulabsolventen in Italien : zwischen

konjunkturellem Aufschwung und strukturellen Schwierigkeiten

El mercado de trabajo de los graduados universitarios en Italia : entre

perspectivas de recuperación y dificultades estructurales

Silvia Ghiselli

\section{OpenEdition}

Journals

Édition électronique

URL : http://journals.openedition.org/formationemploi/7526

DOI : 10.4000/formationemploi.7526

ISSN : 2107-0946

Éditeur

La Documentation française

Édition imprimée

Date de publication : 31 octobre 2019

Pagination : $15-47$

ISSN : 0759-6340

Référence électronique

Silvia Ghiselli, « Le marché du travail des diplômés universitaires en Italie : entre perspectives de reprise et difficultés structurelles », Formation emploi [En ligne], 147 | Juillet-Septembre, mis en ligne le 31 octobre 2019, consulté le 02 mars 2021. URL : http://journals.openedition.org/formationemploi/ 7526 ; DOI : https://doi.org/10.4000/formationemploi.7526 


\title{
Le marché du travail des diplômés universitaires en Italie : entre perspectives de reprise et difficultés structurelles
}

\author{
SILVIA GHISELLI \\ Responsable Bureau des enquêtes et des statistiques, Consorzio Interuniversitario \\ AlmaLaurea, Bologna, Italie
}

Résumé

\begin{abstract}
Le marché du travail des diplômés universitaires en Italie : entre perspectives de reprise et difficultés structurelles

Ce texte examine les tendances les plus récentes du marché du travail des diplômés universitaires, en Italie, et met en exergue les effets de la crise mondiale. Nous présentons d'abord les réformes qui ont caractérisé le système universitaire et le marché du travail au cours des vingt dernières années. Ensuite, nous considérons les débouchés professionnels des diplômés, un an et cinq ans après l'obtention du diplôme, et analysons les facteurs offrant davantage d'opportunités d'emploi aux diplômés et de meilleurs salaires. À cet effet, nous mobilisons deux enquêtes annuelles réalisées par AlmaLaurea, consortium représentant environ 90 \% des diplômés universitaires en Italie.
\end{abstract}

Mots clés : situation du marché du travail, sécurisation des parcours professionnels, politique de l'emploi, mesure pour l'emploi, investissement en formation, politique de l'éducation, enseignement universitaire, insertion professionnelle, disparité régionale, Italie

Abstract

Italy's graduate labour market: possible economic recovery and structural challenges

This paper focuses on the most recent trends in Italy's graduate labour market, highlighting the effects of the global crisis. Firstly, we walk readers through the reforms that have characterised the Italian university system and the domestic labour market over the last 20 years. Secondly, we analyse graduates' employment outcomes, one and five years after graduation, including some in-depth analysis on the elements that offer better employment opportunities and salaries to graduates when they enter the labour market: this analysis is made possible thanks to the annual surveys performed by AlmaLaurea, the Consortium that represents about 90\% of Italy's graduates. 
Keywords: labour market situation, flexicurity, employment policy, employment scheme, investment in training, education policy, university education, transition from school to work, regional disparity, Italy

Journal of Economic Literature: J 24 ; I 23

Traduction : auteure.

\section{Introduction}

La crise mondiale a eu des effets significatifs sur le système productif italien. Au cours des dernières années, on a enregistré de timides signes d'amélioration, mais le tableau est loin de s'être éclairci et les conditions ne sont pas encore réunies pour pouvoir affirmer que la crise et ses conséquences ont été totalement résolues.

En 2018, la croissance de l'économie italienne a enregistré un ralentissement, après la tendance positive des deux années précédentes : le Produit Intérieur Brut (PIB) a augmenté de 0,9\% par rapport au taux de 1,8\% enregistré dans la Zone Euro (ISTAT, 2019a). Dans ce contexte, le marché du travail italien affiche une croissance continue (ISTAT, 2019b) : en 2018, le taux d'emploi dans la tranche d'âge des 20-64 ans est de $63 \%$, en hausse de 0,7 points de pourcentage par rapport à 2017 , et supérieur aux taux enregistrés pendant les années ayant précédé la crise (ISTAT, 2019c).

Les objectifs que l'Italie s'est fixés pour 2020, visant un taux d'emploi de $67 \%$, dans cette tranche d'âge, sont encore loin d'être atteints. Le pays avance à deux vitesses, avec le Centre-Nord qui a déjà dépassé ces objectifs et le Sud qui enregistre un écart de presque vingt points de pourcentage (le taux d'emploi parmi les 20-64 ans n'atteint pas $50 \%)$.

La comparaison à l'échelle européenne (Eurostat, 2019a) met l'accent sur deux éléments : d'une part, l'écart historique, en termes d'emploi, enregistré entre l'Italie et les partenaires européens (au niveau européen, le taux d'emploi des 20-64 ans est de $73,2 \%$ ). D'autre part, l'impact différencié des politiques mises en œuvre par chaque pays membre sur la capacité de réaction des marchés du travail (AlmaLaurea, 2019b) : l'Allemagne, par exemple, a déjà atteint son objectif d'un taux d'emploi de $77 \%$ en 2020, tandis que la France est à 3,7 points d'écart de son objectif de 75 \% (Eurostat, 2019a). "En regardant le modèle européen, l'écart en termes d'emploi en Italie concerne les emplois qualifiés, dans les services publics ou dans les entreprises, pour lesquels un niveau de diplôme élevé est nécessaire" (ISTAT, 2019b).

Dans un marché du travail marqué par des difficultés structurelles, que la crise a de fait contribué à révéler (Banca d'Italia, 2015), le diplôme représente encore une garantie contre le chômage (AlmaLaurea, 2019b) : en Italie, entre 2007 et 2014, le taux de chômage des 15-74 ans a augmenté de 3,5 points de pourcentage chez les diplômés et de 
6,3 points chez les titulaires d'un diplôme de fin d'études secondaires (ISTAT, 2019e). Ce tableau est toutefois à nuancer : "la retombée économique de la scolarité est limitée en Italie, surtout pour les plus jeunes " (Cipollone, Montanaro, Sestito, 2012) et ceci concerne surtout l'entrée des diplômés sur le marché du travail.

Ce résultat n'est pas surprenant, si l'on considère que l'Italie est un pays où les étudiants sont diplômés à un âge moyen plus élevé et où le salaire rémunère mieux certains paramètres, comme l'ancienneté professionnelle, que la possession d'un diplôme de niveau élevé. Parmi les 25-64 ans, en considérant comme base 100 le salaire des titulaires d'un diplôme de fin d'études secondaires, un diplômé universitaire italien perçoit en moyenne 138,5 ; en Italie, cet avantage salarial n'est pas élevé comme dans d'autres pays européens (en moyenne 150,9 ; en France, il est de 154,8), mais il est cependant appréciable et significatif (OCDE, 2018). Pour autant, cela est vrai seulement plusieurs années après l'obtention du diplôme : parmi les travailleurs italiens les plus jeunes (25-34 ans), le diplôme offre seulement un premier, mais partiel, écart de salaire. En considérant comme base 100 le salaire des titulaires d'un diplôme de fin d'études secondaires, un jeune diplômé universitaire italien a un salaire égal à 112,7 ; en moyenne, un jeune diplômé universitaire européen se situe à 133,4 et un diplômé français à 151,6 (OCDE, op. cit.).

L’année 2019 signe le 20e anniversaire du Processus de Bologne, visant à créer un espace européen de l'enseignement supérieur : cela fournit donc une occasion stimulante pour tenter de dresser un bilan de ce qui s'est passé en Italie, au regard du marché du travail des diplômés universitaires. C'est l'objectif de la présente contribution qui, tout en étant principalement descriptive, se place dans le sillon d'une évaluation du rendement des études. Cette évaluation postule, selon une vision économique, que le capital humain est un bien sur lequel investir (Becker, 1964), dont la valeur de l'investissement augmente avec le niveau d'études (Johnes, 1993).

Le choix d'investir dans l'éducation est un choix personnel, qui induit à renoncer à ce dont on pourrait disposer dans l'immédiat, pour obtenir davantage de bénéfices dans le futur (Visco, 2009). Cependant, il est vrai que ce choix, quoique personnel, a un impact social. En effet, le capital humain définit la frontière de la connaissance et de la technologie, avec des retombées sur la productivité globale de l'économie (Cipollone $\&$ Sestito, 2010). Le capital humain est donc considéré un facteur de croissance économique fondamental (Hanushek \& Woessmann, 2009). Ceci est particulièrement vrai dans un contexte de plus en plus complexe et sujet à des évolutions technologiques continues, où la mondialisation et le processus de vieillissement de la population sont inévitables (Cipollone, Montanaro, Sestito, 2012). Dans ce cadre, la crise économique a remis à l'ordre du jour des réflexions importantes. En effet, si le capital humain n'est pas opportunément développé, il peut devenir obsolète et se déprécier, en raison soit du progrès des connaissances, soit de la possible déperdition des connaissances au cours du temps (Praussello, Marenco, 1996). 
Dans ce cadre, la réflexion sur l'Italie adopte des contours particuliers, dans la mesure où le pays souffre encore aujourd'hui d'un retard évident en termes de niveaux de scolarisation, aussi bien parmi la population adulte que chez les jeunes, avec par ailleurs d'importantes disparités territoriales (AlmaLaurea, 2019a) : ainsi, en 2018, le taux des diplômés parmi les 30-34 ans est de 27,8\% par rapport à une moyenne européenne de 40,7\% ; en France, ce taux est de 46,2 \% (Eurostat 2019b). L'écart est dû à l'absence (presque totale) de filières professionnelles non universitaires (niveau 5 - ISCED 2011) et au faible nombre de diplômés universitaires de premier cycle (niveau 6) ; en revanche, en raison du taux élevé de poursuite d'études entre le premier et le second cycles (AlmaLaurea, 2019b), le taux des diplômés d'un master (niveau 7), est conforme aux données européennes (ANVUR, 2018). À cet égard, des préconisations ont été formulées à l'Italie afin d'encourager la recherche, l'innovation, les compétences numériques et les infrastructures et d'accroître la participation à l'enseignement professionnel supérieur (Conseil de l'Union européenne, 2018).

Aux bas niveaux d'éducation sont associés des investissements limités dédiés à l'enseignement de troisième cycle (OCDE, 2018) et à la recherche et développement (Eurostat, 2019c) : "Notre pays qui, dans l'ensemble, par rapport aux bas niveaux d'éducation, se caractérise aussi par un faible investissement en termes de recherche et de développement, ainsi que par une activité modeste en matière de brevets, a donc fondé une part importante de son bienêtre sur des productions dont le contenu en connaissances spécialisées est relativement limité, et qui sont aisément reproductibles ailleurs à moindres coûts" (ISTAT, 2018a).

Tout ceci s'inscrit dans un contexte d'entreprises caractérisé, par ailleurs, par la primauté des petites entreprises, principalement à gestion familiale de type non managérial, par des systèmes d'organisation basés sur un faible recours à des mécanismes incitatifs en matière de salaire et par un faible niveau de formation des entrepreneurs (Bugamelli \& al., 2012). Il est à noter, par ailleurs, que "dans l'activité des petites entreprises, le niveau d'études des entrepreneurs et des employés est associé à des questions de survie, de place sur le marché, de propension à innover et d'adoption de technologies de l'information» (ISTAT, 2018a).

L'analyse des tendances du marché du travail des diplômés, en Italie, doit donc tenir compte du contexte, également normatif, dans lequel les jeunes font leur choix. Ainsi, dans la partie 2 , nous proposons des points de réflexion sur les réformes qui ont caractérisé le système universitaire et le marché du travail italien au cours des vingt dernières années. Dans la partie 3, nous examinons également, dans une approche territoriale et de domaine d'études, les tendances les plus récentes des débouchés sur le marché du travail des diplômés. Dans la partie 4, nous mobilisons certaines analyses sur les facteurs offrant davantage d'opportunités d'emploi aux diplômés et de meilleurs salaires lors de l'accès au marché du travail, avant de conclure. 


\section{Système universitaire et marché du travail : vingt ans de réformes}

Parmi les nombreuses réformes qui ont caractérisé le système universitaire et le marché du travail italien, que nous illustrerons brièvement ci-dessous, nous centrerons notre attention sur ce qui s'est produit au cours des vingt dernières années. En effet, la Réforme du système universitaire italien, qui a eu lieu à la fin des années 90, a de fait jeté les bases de l'actuelle configuration du système d'enseignement de troisième cycle (Vaira, 2011).

\subsection{Un système universitaire marqué par de profondes réformes}

Le système universitaire italien a connu, à la fin des années 90, une profonde réorganisation des cycles d'études (Regini, 2014). Elle a été rendue nécessaire afin de respecter les dispositions du Processus de Bologne, consistant en une réforme des systèmes d'études européens qui visait à créer un espace européen de l'enseignements supérieur (EEES), en facilitant la mobilité des étudiants et des enseignants et en favorisant les possibilités d'emploi.

Par ailleurs, l'Italie, a été l'un des premiers pays européens à réformer ses parcours d'études (Luzzatto, Mangano, Moscati, 2011) : la première Réforme (D.M. n 509/1999) date de 1999 et a été rapidement mise en œuvre dans les universités italiennes. En 2004, la seconde Réforme (D.M. $\mathrm{n}^{\circ}$ 270/2004) a, entre autres, introduit le diploma supplement (un certificat où figurent, conformément aux modèles européens, des indications relatives au curriculum spécifique suivi par l'étudiant, art. 11, alinéa 8) : elle a revu les crédits associés à chaque niveau de diplôme, redéfinissant ainsi les classes de diplôme (clusters de domaines d'études regroupés par des objectifs de formation définis par la loi) ${ }^{1}$.

L'organisation des parcours d'études universitaires italiens, conformément aux dispositions du Processus de Bologne, se base sur trois cycles ou niveaux : un premier cycle, d'une durée de trois ans, sanctionné par le "diplôme universitaire de premier cycle " ou simplement "diplôme universitaire " (qui en France correspond à la " licence ") ; un second cycle, d'une durée de deux ans, à l'issue duquel on obtient le " diplôme universitaire de second cycle " ou " master " (en France " master ") et un troisième cycle qui conduit au « doctorat de recherche » (en France « doctorat»).

Il existe enfin des parcours d'études d'une durée de 5-6 ans, à programmation nationale, auxquels on s'inscrit après l'obtention du diplôme de fin d'études secondaires et à l'issue duquel on obtient un "master à cycle unique " : il s'agit par exemple des parcours de médecine et chirurgie et de chirurgie dentaire, de médecine vétérinaire, de pharmacie.

1. Dans ce cadre, certains domaines d'études, à savoir ceux des Sciences de la formation primaire, ont tardé à réformer leur organisation de l'enseignement, au point que ce n'est qu'à partir de 2016 que les premiers étudiants des parcours réformés ont été diplômés. 
On compte 98 universités en Italie (UniversItaly) : 67 universités d'État, pour 31 universités non étatiques, dont 11 sont en distanciel. On compte également trois écoles polytechniques d'État et six écoles universitaires supérieures, centres de recherche et de formation qui proposent des parcours d'excellence (Ufficio di statistica USTAT, Bureau des statistiques).

\subsection{Un marché du travail entre flexisécurité et lutte contre la précarité}

Le marché du travail italien a connu, lui aussi, de profondes réorganisations au cours des vingt dernières années, qui ont inévitablement conditionné les modalités d'accès et de valorisation économique des travailleurs. La Loi n 196/1997, dite " Pacchetto Treu ", met notamment l'accent sur la "promotion du travail ", comme le titre de la loi même (Biagi, 1997) : cette loi encadre, entre autres, le travail intérimaire, le temps partiel, le contrat de formation - travail $^{2}$ et le contrat d'apprentissage. "Bien qu'encore sous forme embryonnaire, il s'agit de la première mesure sur le marché du travail qui ne s'arrête pas aux mesures passives [...]. C'est la déclinaison italienne des réformes nord-européennes basées sur la flexsecurity " (Massagli, 2018). Quelques années plus tard, la "Riforma Biagi " (Loi $n^{\circ} 30 / 2003$ ), avec un nom évocateur en " matière d'emploi et marché du travail », encadre un éventail de formes contractuelles, dont le travail intérimaire ("somministrazione di lavoro "), le travail intermittent et le travail réparti entre plusieurs opérateurs, le contrat d'insertion, le contrat de travail sur projet et le contrat de travail occasionnel. Elle redéfinit également le contrat d'apprentissage.

Les deux Réformes susmentionnées, qui encadrent de multiples formes contractuelles, s'insèrent de façon générale dans le cadre européen, lequel a défini des stratégies pour l'emploi, en 1997 et en 2000 (Stratégie de Lisbonne), qui ont ensuite conduit à la Stratégie européenne 2020. Comme évoqué plus haut, dans le cadre européen, la tendance est à l'atteinte d'un équilibre social à travers le modèle de la flexsecurity. "En Italie, il existe un écart bien connu entre la dimension de la flexibilité [...] et la dimension de la sécurité sociale, car le système actuel d'indemnités de chômage est complexe, fragmenté et désorganisé, et n'est pas en mesure de couvrir et d'aider tous les chômeurs " (Tridico, 2014). Ces interventions législatives ont accru la flexibilité du travail, surtout à l'entrée : les formes de flexibilité prévues " ont conduit, dans différents cas, à des formes de précarité associées à des segmentations de type contractuel qui sont discriminantes en ce qui concerne la durée (limitée), la couverture d'assu-

2 Le contrat de formation-travail (contratto di formazione - lavoro) visait à promouvoir l'insertion professionnelle des jeunes. Il n'est plus en vigueur, du moins dans le secteur privé. D’une durée maximale de 24 mois (contre 4 ans pour le contrat d'apprentissage, réglementé par la Loi n ${ }^{\circ}$ 196/1997), ce contrat d'alternance accessible aux jeunes de 16 à 32 ans (contre 16 à 24 ou 26 ans pour l'apprentissage) privilégiait la formation pratique en entreprise et prévoyait des baisses de cotisations sociales. Il comportait aussi des heures de formation théorique, mais moins nombreuses que pour un contrat d'apprentissage (de 20 à 130 heures contre 120 au minimum dans le cas de l'apprentissage).. 
rance (sécurité sociale), les droits (absence de mécanismes d'ancienneté, le TFR-Traitement de Fin de Rapport)" (Biggeri, 2006).

Dix années s'écouleront avant que le législateur n'intervienne de nouveau sur la réglementation du marché du travail : la Loi n 92/2012, dite "Legge Fornero ", dénommée " réforme du marché du travail dans une perspective de croissance ", intervient sur de nombreux fronts, avec comme principal objectif de mettre un frein à la précarité qui s'était répandue au cours des années 2000. La loi abroge les contrats d'insertion et d'association en participation ${ }^{3}$, et pénalise de fait toutes les formes contractuelles alternatives au contrat standard, à savoir le travail à durée indéterminée (Massagli, 2018). Deux ans après, le Jobs Act (Loi n $\left.{ }^{\circ} 183 / 2014\right)$ introduit le contrat à durée indéterminée à protection croissante et abroge le contrat de travail sur projet.

Le Jobs Act est également associé à une série de mesures de réduction des cotisations accordées aux employeurs qui embauchent des travailleurs à durée indéterminée. Le plus récent "Decreto Dignità " (D. L. $n^{\circ}$ 87/2018, Loi n 96/2018) vise principalement à endiguer l'augmentation des contrats à durée déterminée, en faisant passer leur durée maximale de 36 à 24 mois, en n'autorisant que quatre prorogations (au lieu de cinq) et en augmentant le coût du travail correspondant.

Les multiples mesures législatives et réglementaires, conjuguées aux nombreuses baisses des cotisations liées à la réduction du coût du travail et au soutien de l'emploi, ont modifié la géographie du marché du travail italien, en influençant les opportunités d'embauche et de croissance professionnelle des travailleurs, jeunes et diplômés universitaires compris. Des interventions qui ont joué un rôle important, notamment pour les petites entreprises, "particulièrement 'sensibles', dans leurs stratégies d'embauche, aux avantages fiscaux" (ISTAT, 2019b).

\section{Les débouchés sur le marché du travail pendant les années de crise}

Les données mentionnées ici sont tirées des deux enquêtes statistiques menées annuellement par le Consorzio Interuniversitario AlmaLaurea (www.almalaurea.it), auquel adhèrent, en juillet 2019, 75 universités représentant environ $90 \%$ du nombre total des diplômés qui sortent chaque année du système universitaire italien.

La première enquête vise à recueillir des informations sur les diplômés, leurs caractéristiques et les expériences de formation acquises au cours des études universitaires. La seconde, qui fait l'objet des réflexions évoquées ci-après, vise à analyser les débouchés

3. Il s'agit d'une forme contractuelle qui prévoit que le salarié reçoive une part des bénéfices de l'entreprise de son employeur en guise de compensation pour son travail. 
sur le marché du travail des diplômés, un an, trois ans et cinq ans après l'obtention du diplôme ; l'intégration des résultats des deux enquêtes permet de tracer, dans une approche longitudinale de type tendance (Bailey, 2006), les trajectoires de formation et d'insertion sur le marché du travail des diplômés, à court et à moyen termes.

La XXIe enquête AlmaLaurea sur les débouchés, la plus récente dont on dispose, a été réalisée en 2018 : l'enquête a concerné plus de 630000 diplômés des 75 universités adhérentes (AlmaLaurea, 2019b). Il s'agit d'une enquête de type recensement, menée grâce à une double méthodologie de relevé, web (CAWI) et téléphonique (CATI). Cette méthodologie concerne tous les diplômés un an après le diplôme et les seuls diplômés de second cycle trois et cinq ans après l'obtention du diplôme. Deux enquêtes ultérieures, menées exclusivement via le web, ont concerné les diplômés de premier cycle, de 2015 et de 2013, n'ayant pas poursuivi d'études vers le second cycle, et qui ont été contactés trois et cinq ans après l'obtention du diplôme. Le taux de réponse est de 78,2 \% chez les diplômés - de premier et de second cycles - un an après l'obtention du diplôme, de 70,4 \% et de 64,5 \% parmi les diplômés de second cycle, trois et cinq ans après l'obtention du diplôme.

Des analyses spécifiques ont été réalisées pour évaluer l'existence de différences structurelles entre les diplômés interviewés et non interviewés. Elles ont révélé quelques rares disparités (inférieures à deux points de pourcentage), qui ne compromettent pas la représentativité globale des résultats. En particulier, parmi les interviewés (indépendamment du type d'études et de l'année du diplôme), le pourcentage des diplômés des groupes ingénierie et médecine et professions de la santé est légèrement supérieur, avec une moindre présence des diplômés du groupe socio-politique. En outre, on note quelques différences par rapport à la répartition territoriale du lieu de résidence : en effet, parmi les interviewés, le pourcentage de diplômés résidant au Nord est plus important, tandis que celui des diplômés résidant au Sud et dans les Îles est inférieur. Il en est de même des diplômés de l'étranger. Pour ces derniers, indépendamment du type d'études, il existe une difficulté objective à les retrouver. Enfin, l'enquête ne met pas en évidence un taux de participation différent selon le genre.

Des analyses ultérieures (Camillo, Conti, Ghiselli, 2011) ont été réalisées pour contrôler la présence d'éventuelles distorsions liées à la combinaison de différentes méthodes de relevé (CAWI et CATI) : les résultats sont rassurants sur la qualité des données relevées et sur celle des réponses fournies, indépendamment du type de relevé utilisé. En effet, les discordances entre les réponses fournies par ceux qui ont participé à un type de relevé et les réponses fournies par l'autre méthode de relevé sont presque toujours faibles (de l'ordre de quelques points de pourcentage).

Bien qu'issue d'un nombre d'universités de plus en plus élevé, la population AlmaLaurea ne représente pas encore la totalité des diplômés italiens : parmi les universités d'État, seul le Politecnico di Milano n'a pas adhéré, et toutes les universités en distanciel sont absentes. Pour à la fois surmonter cette limite, tenir compte de la composante des 
diplômés non interviewés par AlmaLaurea, obtenir des estimations représentatives de la population de référence, et enfin permettre des comparaisons temporelles, les données ont été soumises à une procédure statistique de "redressement ${ }^{4}$.

Bien que le contexte économique soit encore incertain, l'année 2018 a vu une certaine amélioration de la capacité d'absorption du marché du travail, relativement au capital humain formé dans les universités italiennes : le taux d'emploi, les salaires et la cohérence entre le diplôme obtenu et l'emploi occupé sont en hausse par rapport aux années précédentes. Ces tendances sont notamment observées chez ceux qui ont terminé leur parcours d'études plus récemment et ont évité les conséquences liées à l'entrée sur le marché du travail à une période de crise. En effet, même si les données confirment que l'insertion sur le marché du travail et les conditions de travail des diplômés s'améliorent de manière appréciable avec le temps, après l'obtention du diplôme, pour ceux qui ont achevé leur parcours d'études en période de récession, les signes positifs n'ont été enregistrés que récemment.

Ainsi, les opportunités d'emploi de ceux qui accèdent au marché du travail en période de crise économique sont fortement impactées et ont des répercussions même lors de la phase de reprise économique : selon certaines estimations se référant au contexte des États-Unis (Oreopoulos \& al., 2006), la pénalité salariale de ceux qui s'insèrent sur le marché du travail dans des moments de crise, par rapport à ceux qui le font dans des moments plus favorables, est estimée à 80000 dollars, soit une perte de $20 \%$ du revenu individuel qui s'opère en vingt ans environ. Ce résultat confirme d'autres études (Gangl, 2006) portant sur les expériences de perte du travail et de chômage, réalisées dans le cadre européen (pour la Grande-Bretagne Gregory \& Jukes, 2001 ; pour la France et l'Allemagne, Bender \& al., 2002 ; pour certains pays européens, dont l'Italie, Layte \& al., 2000). C'est donc un lourd fardeau (scarring effect), qui risque de conditionner les opportunités d'emploi et de valorisation professionnelle de générations entières.

\footnotetext{
4. Cette méthode permet de réaliser des estimations, sur les diplômés interviewés, qui se rapportent à l'univers de référence, à savoir aux diplômés de chaque année solaire en Italie. Un " poids » est attribué à chaque interviewé (Ardilly, 2006 ; Deming \& Stephan, 1940), de sorte que les distributions relatives aux variables objet du redressement soient, le plus possible, similaires à celles observées dans l'univers de référence. Intuitivement, si par rapport à une variable spécifique, les deux distributions (celle de la population et celle des interviewés) sont identiques, à chaque interviewé est attribué un " poids " égal à 1. Si un interviewé possède des caractéristiques qui sont plus répandues dans la population des diplômés des universités italiennes que dans celle des interviewés d'AlmaLaurea, il lui sera attribué un "poids " proportionnellement plus élevé ; en revanche, à un diplômé qui a des caractéristiques plus répandues parmi les interviewés d'AlmaLaurea que dans la population globale des diplômés des universités italiennes, il lui sera attribué un "poids " proportionnellement moindre. Les variables considérées sont : type d'études, domaine d'études, zone géographique de l'université, zone géographique de résidence au moment du diplôme, toutes croisées avec la variable du genre.
} 
Nous examinons ci-dessous les débouchés professionnels des diplômés, un an et cinq ans après l'obtention du diplôme, en termes de taux d'emploi, de salaire mensuel net, d'efficacité du diplôme par rapport à l'emploi occupé et de typologie de l'activité professionnelle. Soulignons d'emblée une particularité du système universitaire italien, marqué par un taux de poursuite d'études élevé entre le premier et le second cycle d'études, et qui concerne 61,9\% des diplômés de premier cycle de 2017 interviewés un an après l'obtention du diplôme. Ce phénomène, bien qu'en hausse ces dernières années, caractérise, depuis dix ans, plus de la moitié des diplômés de premier cycle. Nous jugeons donc opportun de restreindre l'analyse aux diplômés de premier cycle qui, après l'obtention du diplôme, ne se sont pas inscrits à un autre cycle d'études (soit $37,2 \%$ des diplômés de premier cycle).

\subsection{Un taux d'emploi qui s'améliore légèrement, mais un écart Nord/ Sud qui persiste}

En 2018, le taux d'emploi, un an après l'obtention du diplôme, est de 72,1 \% chez les diplômés de premier cycle, et de 69,4 \% pour les diplômés de second cycle (Figure 1). Le taux d'emploi tend à s'améliorer ces quatre dernières années (par rapport à 2014, année du minimum " historique " pour les diplômés universitaires un an après l'obtention du diplôme, $+6,4$ points de pourcentage pour les diplômés de premier cycle et $+4,2$ points pour les diplômés de second cycle). Il s'agit de signes positifs qui ne sont toutefois pas encore en mesure de compenser la diminution importante du taux d'emploi observée entre 2008 et 2014.

Malgré les inévitables difficultés rencontrées par ceux qui sont entrés sur le marché du travail au moment le plus critique de la crise, cinq ans après l'obtention du diplôme, les diplômés ont des taux d'emploi plus élevés et la tendance est à l'amélioration : le taux d'emploi est de 88,6 \% chez les diplômés de premier cycle et de 85,5 \% parmi les diplômés de second cycle (Figure 1). Ces taux sont en hausse, par rapport aux valeurs minimums enregistrées en 2015 , respectivement de 3,0 et de 0,8 points de pourcentage. Mais dans ce cas aussi, ces signes positifs se manifestent après des années de baisse importante du taux d'emploi.

Les différences territoriales sont significatives, en particulier un an après l'obtention du diplôme : chez les diplômés de premier cycle, l'écart Nord-Sud est de 18,9 points de pourcentage, et de 19,2 points chez les diplômés de second cycle. L'écart Nord-Sud, quoiqu'en diminution, reste également important cinq ans après l'obtention du diplôme (14,1 points de pourcentage chez les diplômés de premier cycle, 9,8 points chez les diplômés de second cycle). 
Figure 1. Diplômés des années 2007-2017 interviewés un an et cinq ans après l'obtention du diplôme : taux d'emploi par type d'études.

Années d'enquête 2008-2018 (valeur en \%)
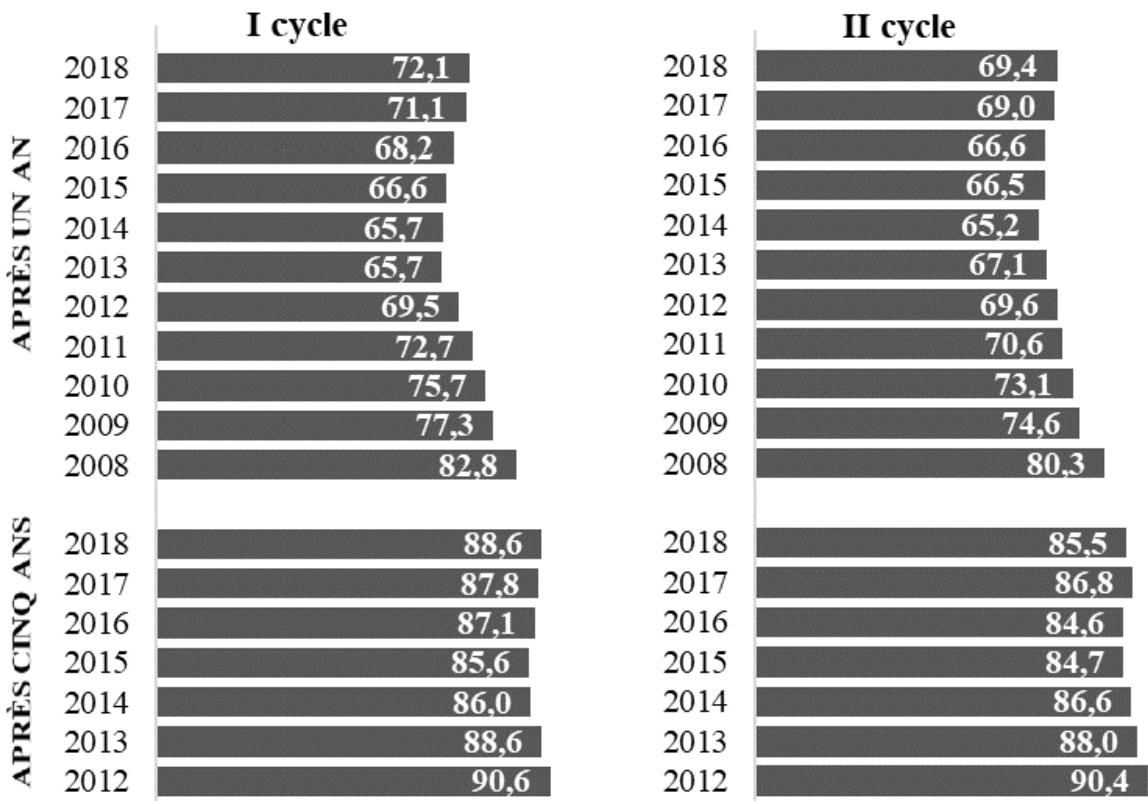

Note : pour le premier cycle, nous avons tenu compte uniquement des diplômés non inscrits à un autre cycle d'études. Source : AlmaLaurea (2019b). 
On note des différences sensibles selon le domaine d'études suivi : en effet, à la fois parmi les diplômés de premier cycle et parmi ceux de second cycle, les domaines d'études les plus favorisés sont économie-statistique, ingénierie, médecine et professions de la santé, pharmacie, sciences et études vétérinaires.

À l'inverse, les domaines d'études géo-biologie, droit, lettres et psychologie connaissent quelques difficultés en termes d'insertion sur le marché du travail. Soulignons qu'il ne s'agit pas exclusivement des sciences humaines, contrairement à ce que l'on aurait pu croire. Nous renvoyons au point 3.1 pour une analyse plus fouillée.

\subsection{Les salaires varient selon les domaines d'études, mais le clivage Nord/Sud demeure}

En 2018, le salaire mensuel net un an après le diplôme est en moyenne d'environ 1169 euros chez les diplômés de premier cycle et de 1232 euros chez les diplômés de second cycle (Figure 2). Dans un contexte caractérisé par une bonne stabilité des prix à la consommation (ISTAT, 2019f), les salaires effectifs un an après l'obtention du diplôme sont en hausse par rapport à $2014:+13,4 \%$ pour les diplômés de premier cycle, $+14,1 \%$ pour les diplômés de second cycle. Toutefois, là aussi, la hausse ne parvient pas encore à compenser l'importante perte de salaire enregistrée pendant la période la plus critique (2008-2014).

Cinq ans après l'obtention du diplôme, le salaire mensuel net est en hausse et est d'environ 1418 euros pour les diplômés de premier cycle et de 1459 euros pour ceux de second cycle (Figure 2). L'augmentation généralisée des salaires, enregistrée ces dernières années, même si elle ne compense pas la perte de salaire de la période précédente, réduit toutefois l'écart par rapport à 2012.

En termes de salaire, l'écart territorial demeure significatif, et contrairement aux chiffres enregistrés pour le taux d'emploi, il ne se réduit pas entre un et cinq ans après l'obtention du diplôme. En effet, tant parmi les diplômés de premier cycle, que parmi ceux de second cycle, les salaires des actifs du Nord sont toujours supérieurs (d'au moins $20 \%$ ) à ceux des actifs du Sud. 
Figure 2. Diplômés des années 2007-2017 interviewés un an et cinq ans après l'obtention du diplôme : salaire mensuel net par type d'études. Années d'enquête 2008-2018 (valeurs réévaluées sur la base des indices Istat des prix à la consommation ; valeurs moyennes en euros)
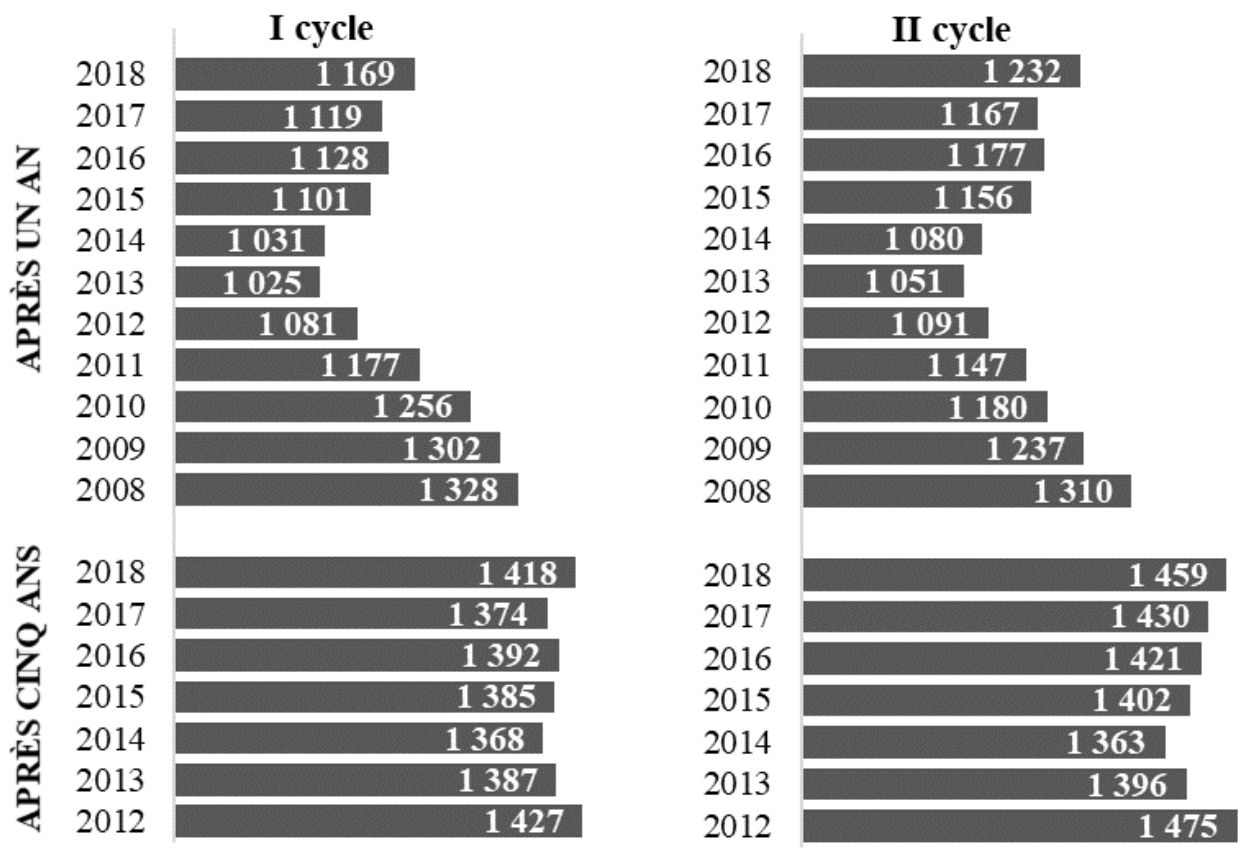

Note : pour le premier cycle, nous avons tenu compte uniquement des diplômés non inscrits à un autre cycle d'études. Source : AlmaLaurea (2019b). 
Les différences par domaine d'études sont significatives en termes de salaire : les domaines les plus favorisés sont l'ingénierie, la médecine et les professions de la santé et les sciences, tandis que la valorisation économique des filières enseignement et lettres est plus modeste. Précisons toutefois que de nombreux facteurs ont une influence sur ce résultat, comme par exemple l'inégale diffusion du travail à temps partiel. Nous renvoyons au point 3.2 pour des analyses sur ce point.

\subsection{Quelle efficacité du diplôme par rapport à l'emploi occupé ?}

Différentes études soulignent les difficultés rencontrées par les diplômés pour trouver un emploi correspondant à leur diplôme ; une situation qui, outre qu'elle persiste dans le temps (Cappelli, 2015), a des effets négatifs sur les salaires et sur la satisfaction vis-à-vis de l'emploi occupé (McGuinness \& Sloane, 2011). Il s’agit de résultats qui mettent en question les investissements individuels et collectifs dans les systèmes d'études (McGuinness, Whelan, Bergin, 2016).

Les données d'AlmaLaurea montrent que pour plus de la moitié des diplômés qui occupent un emploi un an après le diplôme, le diplôme universitaire s'avère " très efficace ou efficace $"^{5}: 56,3 \%$ chez les diplômés de premier cycle et 59,0 \% parmi les diplômés de second cycle (Figure 3). Par rapport à l'enquête de 2014, on note, en 2018, une amélioration de 9,0 points de pourcentage pour les diplômés de premier cycle et de 5,5 points pour les diplômés de second cycle : c'est-à-dire que l'enquête 2018 a confirmé un retour aux valeurs constatées en 2008 .

\footnotetext{
5. L'indicateur d'efficacité du diplôme combine deux questions inhérentes à l'utilisation, dans l'emploi occupé, des compétences acquises à l'université et la nécessité (formelle et substantielle) du diplôme dans l'activité professionnelle exercée. Les niveaux d'efficacité indiqués sont au nombre de cinq :

- très efficace : diplôme exigé par la loi ou de fait nécessaire, et forte utilisation des compétences universitaires acquises ;

- efficace : diplôme non exigé par la loi, mais néanmoins utile, et forte utilisation des compétences, ou diplôme exigé par la loi et faible utilisation des compétences ;

- assez efficace : diplôme non exigé par la loi, mais de fait nécessaire ou utile, et faible utilisation des compétences ;

- peu efficace : diplôme ni exigé par la loi ni utile, et faible utilisation des compétences, ou diplôme non exigé, mais utile, et aucune utilisation des compétences ;

- pas du tout efficace : diplôme non exigé par la loi ni utile, et aucune utilisation des compétences.
} 
Figure 3. Diplômés des années 2007-2017 interviewés un an et cinq ans après l'obtention du diplôme : efficacité du diplôme par type d'études. Années d'enquête 2008-2018 (valeur en \%)
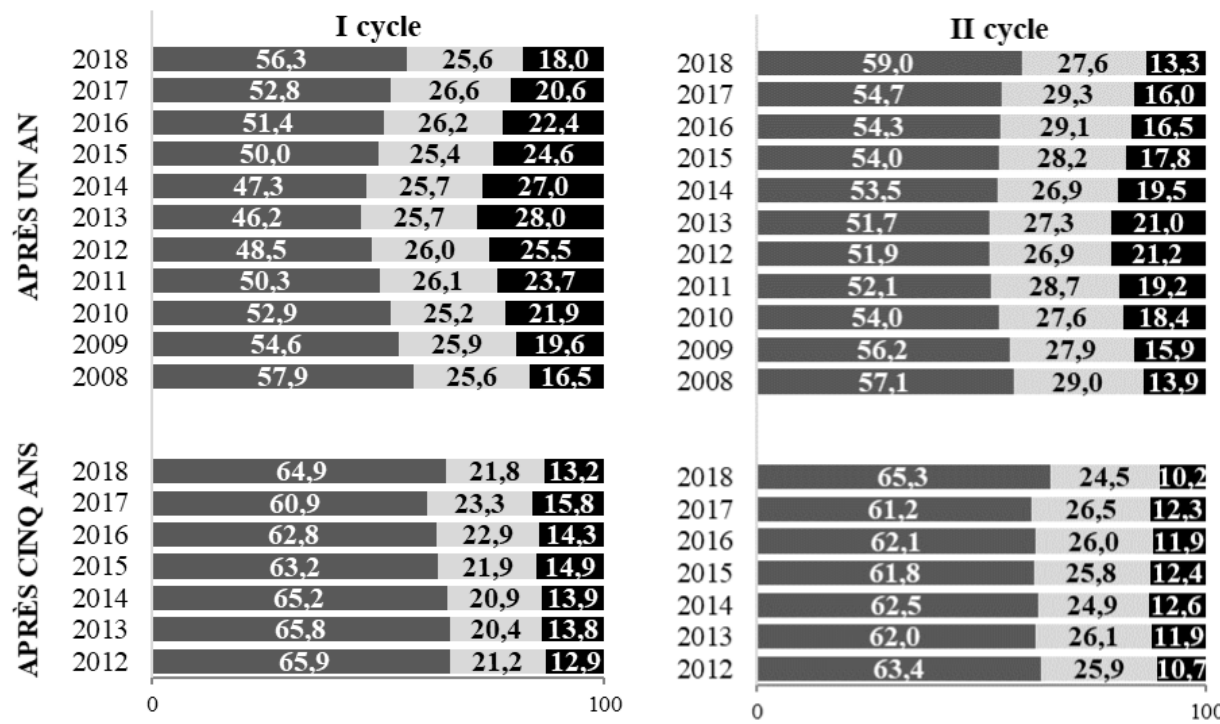

très efficace/efficace assez efficace

- peu/pas du tout efficace

Note : pour le premier cycle, nous avons tenu compte uniquement des diplômés non inscrits à un autre cycle d'études. Source : AlmaLaurea (2019b).

Après cinq ans, les niveaux d'efficacité (modalité " très efficace ou efficace ") augmentent pour atteindre 64,9\% et 65,3\% des actifs de premier et de second cycles (Figure 3). Ces valeurs enregistrent une hausse significative en 2018, au point d'égaler les niveaux d'efficacité de 2012.

De tous les indicateurs examinés, l'efficacité du diplôme est celui qui comporte les plus faibles disparités en termes territoriaux. Ce résultat intéressant est en partie lié à la diffusion hétérogène des formes contractuelles, comme on le verra plus loin, et au poids différent des actifs dans les secteurs public/privé.

Les disparités par domaine d'études sont importantes. Elles dépendent, en général, de la diversité des parcours d'études (professionnel ou généraliste). Ainsi, chez les diplômés des parcours à cycle unique, on observe une meilleure efficacité du diplôme. À l'inverse, pour les diplômés en lettres et socio-politique, on note une plus grande inadéquation entre le diplôme obtenu et l'emploi occupé. 


\subsection{L'activité professionnelle se caractérise par de fortes disparités territoriales}

L'analyse de la typologie de l'activité professionnelle permet de dresser un tableau étroitement lié aux mesures législatives qui se sont succédé au cours du temps (ISTAT, 2019b), mais aussi aux tendances du marché du travail.

Un an après l'obtention du diplôme, la forme contractuelle la plus répandue parmi les actifs est le contrat non standard (principalement des contrats de travail salarié à durée déterminée), qui concerne 39,9\% des diplômés de premier cycle et 35,8 \% de ceux de second cycle (Tableau 1). Le contrat de travail salarié à durée indéterminée concerne $24,5 \%$ des actifs de premier cycle et $24,6 \%$ des actifs de second cycle. Les activités indépendantes (professions libérales, entrepreneurs...) sont également répandues et concernent 13,7 \% des diplômés de premier cycle et 10,9 \% des diplômés de second cycle.

La comparaison avec les données des années précédentes révèle des tendances pas toujours linéaires, par ailleurs difficiles à interpréter étant donné la multitude de facteurs qui en déterminent le résultat : les mesures législatives, le fait d'avoir, parmi les actifs, des diplômés embauchés à différentes périodes, et la crise économique qui a eu une incidence variable sur les marchés du travail. Par rapport à l'enquête de 2008, on note une forte augmentation du travail non standard, en hausse de 16,0 points de pourcentage chez les diplômés de premier cycle et de 14,5 points chez ceux de second cycle. Ceci confirme d'ailleurs les dernières tendances du marché du travail en Italie (ISTAT 2019b). En revanche, le travail salarié à durée indéterminée a diminué par rapport à 2008, de 17,3 points de pourcentage chez les diplômés de premier cycle et de 6,9 points chez ceux de second cycle.

Cinq ans après l'obtention du diplôme, la forme contractuelle la plus répandue est le contrat de travail salarié à durée indéterminée. Il concerne 58,7 \% des diplômés de premier cycle et 52,9 \% des diplômés de second cycle. La part de ceux qui sont embauchés sous contrat non standard est en nette diminution (cinq ans après l'obtention du diplôme, $18,8 \%$ des diplômés de premier cycle et 18,4 \% de ceux de second cycle). Le travail indépendant se stabilise à 10,9\% chez les diplômés de premier cycle et à $21,1 \%$ chez les diplômés de second cycle (Tableau 1) ; les formes de travail indépendant sont plus répandues chez les diplômés de second cycle en raison de leur plus grande propension à s'engager dans des activités professionnelles libérales, en particulier chez les diplômés à cycle unique. La comparaison avec les données des années précédentes montre, également chez les diplômés cinq ans après l'obtention du diplôme, une augmentation du travail non standard (par rapport aux données de $2012:+9,0$ points pour les diplômés de premier cycle et $+3,0$ pour les diplômés de second cycle).

Les disparités territoriales s'avèrent marquées et dépendent étroitement du tissu productif local. Parmi les actifs du Nord, les contrats de travail salarié à durée indéterminée sont plus répandus, et parmi ceux qui occupent un emploi un an après le diplôme, les contrats 
de formation ${ }^{6}$ sont assez répandus. Il s'agit de contrats que les entreprises proposent en guise de première forme d'insertion, notamment pour des raisons liées aux limites d'âge imposées par la loi. Au Sud, en revanche, les activités indépendantes sont plus répandues, comme une réponse des diplômés aux difficultés d'insertion sur le marché du travail.

Tableau 1. Diplômés des années 2007-2017 interviewés un an et cinq ans après l'obtention du diplôme : typologie de l'activité professionnelle par type d'études. Années d'enquête 2008-2018 (valeurs en \%)

\begin{tabular}{|c|c|c|c|c|c|c|c|c|c|c|c|c|c|c|c|c|}
\hline & \multicolumn{2}{|c|}{ Indépendant } & \multicolumn{2}{|c|}{$\begin{array}{c}\text { Durée } \\
\text { indéter- } \\
\text { minée }\end{array}$} & \multicolumn{2}{|c|}{$\begin{array}{c}\text { Contrats } \\
\text { de } \\
\text { formation }\end{array}$} & \multicolumn{2}{|c|}{$\begin{array}{c}\text { Non } \\
\text { standard }\end{array}$} & \multicolumn{2}{|c|}{$\begin{array}{l}\text { Para- } \\
\text { subordonné }\end{array}$} & \multicolumn{2}{|c|}{$\begin{array}{c}\text { Autres } \\
\text { autonomes }\end{array}$} & \multicolumn{2}{|c|}{$\begin{array}{c}\text { Sans } \\
\text { contrat }\end{array}$} & \multicolumn{2}{|c|}{$\begin{array}{l}\text { Ne répond } \\
\text { pas }\end{array}$} \\
\hline & $I$ & II & I & II & I & II & I & II & I & II & $\overline{\mathrm{I}}$ & II & I & II & $I$ & $\mathrm{II}$ \\
\hline & cycle & & cycle & cycle & cycle & cycle & cycle & cycle & cycle & cycle & cycle & cycle & cycle & cycle & cycle & cycle \\
\hline \multicolumn{17}{|c|}{ APRÈS UN AN } \\
\hline 2018 & 13,7 & 10,9 & 24,5 & 24,6 & 11,3 & 15,4 & 39,9 & 35,8 & 2,1 & 2,9 & 4,1 & 5,5 & 3,9 & 4,4 & 0,4 & 0,4 \\
\hline 2017 & 12,9 & 10,5 & 23,5 & 24,0 & 10,0 & 13,8 & 38,1 & 34,4 & 2,8 & 3,1 & 5,6 & 6,8 & 6,5 & 6,8 & 0,6 & 0,6 \\
\hline 201 & 14,4 & 11,7 & 29,0 & 30,9 & 8,9 & 12,4 & 32,9 & 8,2 & 2 , & 3,3 & & 6,8 & 5,5 & 6,2 & 0,4 & 0,5 \\
\hline 2015 & 15,4 & 11,5 & 27,7 & 26,6 & 8,8 & 12,9 & 31,3 & 30,8 & 4,8 & 6,1 & 5 . & 6,0 & 6,0 & 5,6 & 0,5 & 0,4 \\
\hline 2014 & 14,7 & 11,2 & 24,1 & 22,4 & 9,9 & 13,6 & 29,8 & 27,9 & 7,2 & 9,2 & 6,4 & 7,7 & 7,5 & 7,5 & 0,4 & 0,5 \\
\hline 2013 & 14,4 & 11,0 & 26,5 & 23,3 & 9,4 & 12,7 & 27,2 & 26,2 & 7,1 & 9,6 & 6 & 7,9 & 8,3 & 9,1 & 3 & 0,3 \\
\hline 2012 & 12,1 & 9,3 & 28,9 & 23,7 & 8,6 & 12,0 & 27,2 & 26,4 & 9,1 & 12,4 & 8 & 8,0 & 7,1 & 7,9 & 0,3 & 0,3 \\
\hline 201 & 10,9 & 9,2 & 31,3 & 24,1 & 8,3 & 11,9 & 27,7 & 25,7 & 9,8 & 14,0 & & 7,5 & 6,4 & 7,3 & 0,2 & 0,3 \\
\hline 2010 & 11,3 & 9,1 & 34,5 & 24,6 & 6,9 & 10,1 & 27,3 & 27,1 & 9,1 & 14,3 & 4,7 & 7,4 & 5,9 & 7,1 & 0,2 & 0,3 \\
\hline 2009 & 9,9 & 8,9 & 39,2 & 28,4 & 7,9 & 11,4 & 25,0 & 25,5 & 8,7 & 14,3 & 4,1 & 6,0 & 5,1 & 5,3 & 0,1 & 0,2 \\
\hline 2008 & 9,4 & 8,5 & 41,8 & 31,5 & 8,5 & 14,2 & 23,9 & 21,3 & 9,0 & 14,0 & 3,5 & 6,1 & 3,7 & 4,1 & 0,2 & 0,3 \\
\hline \multicolumn{17}{|c|}{ APRÈS CINQ ANS } \\
\hline 2018 & 10,9 & 21,1 & 58,7 & 52,9 & 4,2 & 2,2 & 18,8 & 18,4 & 1,5 & 1,5 & 3,0 & 2,3 & 1,7 & 1,2 & 1,1 & 0,4 \\
\hline 2017 & 12,7 & 21,1 & 56,0 & 50,3 & 4,1 & 2,2 & 17,9 & 18,6 & 1,7 & 1,9 & & 3,2 & 2,3 & 1,9 & 1,9 & 0,7 \\
\hline 2016 & 13,7 & 23,7 & 60,7 & 52,5 & 3,9 & 2,2 & 14,7 & 15,5 & 1,4 & 2,0 & 2,8 & 2,5 & 1,8 & 1,3 & 1,1 & 0,4 \\
\hline 2015 & 14,5 & 24,3 & 57,9 & 49,4 & 4,4 & 3,2 & 13,6 & 15,1 & 3,2 & 3,9 & 3,4 & 2,4 & 2,1 & 1,4 & 0,9 & 0,2 \\
\hline 2014 & 14,0 & 3,4 & 59,6 & 46,4 & 4,6 & 3,6 & 12,0 & 16,7 & 3,5 & 5,1 & 2,9 & 2,9 & 2,0 & 1,6 & 1,3 & 0,3 \\
\hline 2013 & 11,6 & 22,8 & 66,8 & 49,4 & 3,8 & 3,4 & 10,0 & 14,8 & 3,1 & 5,2 & 2,7 & 2,7 & 1,9 & 1,5 & 0,1 & 0,2 \\
\hline 2012 & 10,4 & 19,9 & 68,4 & 50,9 & 3,4 & 2,8 & 9,8 & 15,4 & 4,1 & 6,3 & 2,5 & 3,2 & 1,3 & 1,3 & 0,1 & 0,2 \\
\hline
\end{tabular}

Note : pour le premier cycle, nous avons tenu compte uniquement des diplômés non inscrits à un autre cycle d'études. Source : AlmaLaurea (2019b).

6. Il s'agit de contrats de travail salarié, surtout d'apprentissage, avec un contenu mixte de formation et d'emploi. Ils s'adressent aux jeunes de 15 à 29 ans (le contrat d'insertion, dans des cas particuliers, peut être prolongé au-delà de 29 ans). 
S'agissant du domaine d'études, on enregistre des disparités qui dépendent de nombreux facteurs, entre autres le poids différent des diplômés qui intègrent les secteurs public/privé, les choix individuels liés au démarrage de la profession libérale, la poursuite ou non d'activités professionnelles ayant débuté en cours d'études. Par exemple, parmi ceux qui s'insèrent dans l'enseignement (il s'agit notamment des diplômés des parcours en sciences humains), les contrats non standards sont relativement plus répandus, même cinq ans après l'obtention du diplôme, et ce, en raison du blocage du recrutement qui a longtemps empêché, en Italie, la stabilisation contractuelle des enseignants du secteur public.

\section{Les débouchés varient selon plusieurs facteurs}

Afin d'analyser, globalement, les multiples facteurs qui ont une influence sur la probabilité de trouver un emploi et sur le salaire des diplômés, nous avons adopté deux modèles de régression, respectivement logistique et linéaire, qui sont illustrés ci-dessous. Le but de l'analyse est purement descriptif. Il s'agit d'identifier les associations entre les variables considérées. Nous avons pris en compte les diplômés de 2017, contactés un an après l'obtention du diplôme ${ }^{7}$ : ce choix découle de la nécessité, d'une part, d'évaluer comment se caractérise la première phase d'insertion sur le marché du travail, et d'autre part, de contrôler les facteurs qui sont susceptibles d'avoir un impact. En effet, entre un et cinq ans après l'obtention du diplôme, les diplômés acquièrent des expériences, professionnelles et de formation qui sont plus difficiles à appréhender avec des interviews réalisées annuellement ; en outre, au fur et à mesure que l'on s'éloigne de la date d'obtention du diplôme, l'effet des expériences acquises au cours des études universitaires (ou précédemment) s'amenuise.

Les analyses ont tenu compte des nombreux facteurs liés aux aspects socio-démographiques, au diplôme universitaire, aux expériences et aux compétences acquises en cours d'études, aux aspirations et inclinaisons déclarées par les diplômés à la veille de la fin des études. Dans le modèle utilisé pour le salaire, étant donné l'approche descriptive adoptée, et pour une analyse plus structurée, nous avons décidé de prendre également en compte certains facteurs concomitants, relatifs aux caractéristiques de l'emploi occupé ${ }^{8}$.

7. Les modèles ne tiennent pas compte de ceux qui travaillaient déjà au moment de l'obtention du diplôme, ni des résidants à l'étranger, des diplômés des filières défense et sécurité, ni des diplômés du cycle d'études en Sciences de la formation primaire suivi avant la réforme, étant donné la particularité des parcours d'études et leur faible nombre.

8. Les analyses descriptives des variables insérées dans les modèles sont disponibles sur demande. 


\subsection{Des disparités d'emploi selon les domaines d'études, le diplôme et la région}

Le Tableau 2 présente les résultats du modèle relatif à l'évaluation de la probabilité de trouver un emploi un an après le diplôme ; nous décrivons ci-après quelques-uns des facteurs intéressants.

Tout d'abord, le domaine d'études a un effet sur les opportunités d'emploi des diplômés : toutes choses égales par ailleurs, les diplômés des groupes ingénierie, sciences, chimie, pharmacologie, médecine et professions de la santé s'avèrent plus favorisés. En revanche, les diplômés en psychologie, droit et lettres le sont moins. Par ailleurs, les diplômes de second cycle ont davantage d'opportunités d'emploi un an après le diplôme : en effet, par rapport aux diplômés de premier cycle, les diplômés titulaires d'un master comportant deux ans d'études ont 34,7\% de probabilité en plus de trouver un emploi et les diplômés d'un master à cycle unique ont 14,7\% de probabilité en plus (dans cette analyse, nous avons jugé opportun de distinguer les parcours de second cycle en raison de l'approche différente à l'égard du marché du travail adoptée par les diplômés à cycle unique, nettement plus enclins à accéder aux professions libérales). Ce résultat - bien qu'il faille l'interpréter avec précaution dans la mesure où l'on compare des populations profondément différentes en termes de parcours de formation et de perspectives professionnelles et d'études - est cohérent avec le domaine de recherche en termes d'évaluation du rendement du capital humain.

Les différences de genre, et surtout les disparités territoriales, demeurent significatives. Elles montrent, ceteris paribus, un meilleur positionnement des hommes (16,1\% de probabilité en plus de trouver un emploi par rapport aux femmes) et de ceux qui résident ou qui ont étudié au Nord (s'agissant du lieu de résidence, + 41,2\% de probabilité de trouver un emploi par rapport à ceux qui résident au Sud ; s'agissant de la répartition géographique des études, $+76,6 \%$ de probabilité de trouver un emploi par rapport à ceux qui ont étudié au Sud).

Le contexte socio-culturel d'origine a des effets sur les débouchés des diplômés, quoique l'analyse révèle une influence limitée : les diplômés issus de familles où au moins un des parents est diplômé de l'université ont une moindre probabilité d'être en emploi (- 10,0 \%) que ceux dont les parents ne possèdent pas de diplôme universitaire. Le résultat laisse supposer que le cadre familial, outre le fait qu'il exerce une influence sur le choix de formation des jeunes, conditionne aussi les débouchés (Bernardi \& Ballarino, 2016, Galeazzi \& Ghiselli 2016), permettant aux diplômés de différer l'accès au marché du travail, dans l'attente d'un meilleur emploi. 
Tableau 2. Diplômés de premier et de second cycles de l'année 2017, interviewés un an après l'obtention du diplôme : modèle de régression logistique pour l'évaluation de la probabilité de trouver un emploi. Année d'enquête 2018

\begin{tabular}{|c|c|c|c|}
\hline & b & Erreur-type & $\operatorname{Exp}(b)$ \\
\hline \multicolumn{4}{|l|}{ Genre (femmes $=0$ ) } \\
\hline Hommes & 0,149 & 0,017 & 1,161 \\
\hline \multicolumn{4}{|c|}{ Au moins un parent diplômé de l'université (non = 0) } \\
\hline oui & $-0,106$ & 0,017 & 0,900 \\
\hline \multicolumn{4}{|c|}{ Répartition géographique de la résidence (Sud = 0) } \\
\hline Nord & 0,345 & 0,030 & 1,412 \\
\hline Centre & 0,232 & 0,029 & 1,261 \\
\hline \multicolumn{4}{|l|}{ Type de cours (premier cycle $=0$ ) } \\
\hline Master de deux ans $(B A C+2)$ & 0,298 & 0,024 & 1,347 \\
\hline Master à cycle unique & 0,137 & 0,029 & 1,147 \\
\hline \multicolumn{4}{|c|}{ Domaine d'études (Socio-politique $=0$ ) } \\
\hline Agriculture et études vétérinaires & 0,515 & 0,057 & 1,674 \\
\hline Architecture & 0,346 & 0,041 & 1,414 \\
\hline Chimie et pharmacologie & 1,118 & 0,051 & 3,058 \\
\hline Économie et statistique & 0,611 & 0,033 & 1,842 \\
\hline Éducation physique & 0,238 & 0,074 & 1,269 \\
\hline Géobiologie & 0,191 & 0,045 & 1,211 \\
\hline Droit & $-0,305$ & 0,042 & 0,737 \\
\hline Ingénierie & 1,480 & 0,041 & 4,393 \\
\hline Enseignement & 0,629 & 0,050 & 1,876 \\
\hline Lettres & $-0,183$ & 0,039 & 0,833 \\
\hline Linguistique & 0,264 & 0,042 & 1,302 \\
\hline Médecine et professions de la santé & 1,164 & 0,035 & 3,204 \\
\hline Psychologie & $-0,867$ & 0,048 & 0,420 \\
\hline Sciences & 1,445 & 0,066 & 4,242 \\
\hline \multicolumn{4}{|c|}{ Répartition géographique de l'université (Sud = 0) } \\
\hline Nord & 0,569 & 0,030 & 1,766 \\
\hline Centre & 0,294 & 0,028 & 1,342 \\
\hline Âge à l'obtention du diplôme & $-0,058$ & 0,003 & 0,944 \\
\hline \multicolumn{4}{|c|}{$\begin{array}{l}\text { Régularité des études (prolongation de la durée des études : } 2 \text { ans } \\
\text { et plus }=0 \text { ) }\end{array}$} \\
\hline Moins d'un an de retard & 0,118 & 0,022 & 1,125 \\
\hline \multicolumn{4}{|l|}{ Stage universitaire $($ non $=0$ ) } \\
\hline oui & 0,087 & 0,017 & 1,091 \\
\hline \multicolumn{4}{|c|}{ Travail pendant les études (aucun emploi $=0$ ) } \\
\hline Travailleur-étudiant & 0,502 & 0,054 & 1,651 \\
\hline Étudiant-travailleur & 0,330 & 0,016 & 1,391 \\
\hline
\end{tabular}




\begin{tabular}{|c|c|c|c|}
\hline \multicolumn{4}{|c|}{ Études à l'étranger (aucune expérience $=0$ ) } \\
\hline Erasmus - un autre programme de l'UE & 0,120 & 0,025 & 1,127 \\
\hline Autre expérience & 0,194 & 0,037 & 1,214 \\
\hline \multicolumn{4}{|c|}{ Nombre d'outils informatiques connus (au plus $2=0$ ) } \\
\hline 3 ou 4 outils & 0,140 & 0,025 & 1,151 \\
\hline 5 outils ou plus & 0,232 & 0,022 & 1,261 \\
\hline \multicolumn{4}{|c|}{ Intention de continuer les études (oui $=0$ ) } \\
\hline non & 0,319 & 0,017 & 1,376 \\
\hline \multicolumn{4}{|l|}{ Disponibilité à voyager $(\mathrm{non}=0)$} \\
\hline oui & 0,142 & 0,051 & 1,152 \\
\hline \multicolumn{4}{|c|}{ Attentes : possibilité de carrière $($ non $=0$ ) } \\
\hline oui & 0,077 & 0,020 & 1,080 \\
\hline \multicolumn{4}{|c|}{ Attentes : acquisition de professionnalisme $($ non $=0$ ) } \\
\hline oui & 0,152 & 0,023 & 1,164 \\
\hline \multicolumn{4}{|c|}{ Attentes: stabilité/sécurité de l'emploi (non = 0) } \\
\hline oui & $-0,089$ & 0,020 & 0,915 \\
\hline \multicolumn{4}{|c|}{$\begin{array}{l}\text { Attentes: correspondance de l'emploi avec les intérêts culturels } \\
(\text { non }=0)\end{array}$} \\
\hline oui & $-0,068$ & 0,017 & 0,934 \\
\hline \multicolumn{4}{|c|}{ Attentes : flexibilité des horaires de travail $($ non $=0)$} \\
\hline oui & $-0,118$ & 0,018 & 0,889 \\
\hline Constante & 0,104 & 0,104 & 1,109 \\
\hline
\end{tabular}

Pour chaque variable, nous avons considéré une modalité de référence (indiquée entre parenthèses près du nom de la variable) par rapport à laquelle sont calculés tous les coefficients $b$, et les erreurs-types des autres modalités : des coefficients supérieurs à 0 indiquent un effet positif exercé sur la probabilité de trouver un emploi, des coefficients inférieurs à 0 un effet négatif. Pour faciliter la lecture des coefficients, il est possible de faire référence à exp (b) : par exemple, en ce qui concerne la variable du genre, on remarque que les hommes ont 16,1\% de probabilité en plus de trouver un emploi par rapport aux femmes.

Note : taux de prédiction correcte égal à $67,1 \% ; \mathrm{N}=86647 ; \mathrm{R} 2$ Nagelkerke $=0,199$.

Tous les paramètres sont significatifs à $1 \%(p<0,01)$.

Source : AlmaLaurea (2019b). 
Toutes choses égales par ailleurs, l'âge au moment du diplôme a une incidence négative (- 5,6 \% pour chaque année supplémentaire) sur la probabilité de trouver un emploi un an après l'obtention du diplôme. Ceci est vraisemblablement lié au fait que ceux qui entrent sur le marché du travail précocement ont des perspectives et des disponibilités, même contractuelles, plus attrayantes aux yeux des employeurs.

Les expériences de travail, tout comme certaines compétences acquises au cours des études universitaires, exercent un effet positif sur les possibilités d'emploi. En effet, toutes choses égales par ailleurs, les travailleurs-étudiants (à savoir ceux qui ont acquis des expériences de travail continu et à temps plein pendant au moins la moitié de la durée de leurs études) ont 65,1\% de probabilité en plus de trouver un emploi que les diplômés qui parviennent au diplôme sans aucune expérience professionnelle ; les étudiants-travailleurs (à savoir ceux qui ont eu d'autres types d'expériences de travail) ont 39,1\% de probabilité en plus de trouver un emploi par rapport à ceux qui n'ont acquis aucune expérience professionnelle. Ceux qui ont effectué un stage universitaire ont, ceteris paribus, 9,1\% de probabilité en plus de trouver un emploi un an après l'obtention du diplôme par rapport à ceux qui n'ont pas effectué de stage. Ce résultat est en cohérence avec des études analogues réalisées dans le cadre français (Lopez \& Sulzer, 2016).

De même, ceux qui ont connu une période d'études à l'étranger au cours des études universitaires ont davantage de probabilité de trouver un emploi que ceux qui n'ont jamais effectué de séjour à l'étranger : par exemple, parmi ceux qui ont acquis cette expérience dans le cadre d'un programme de l'Union européenne, la probabilité est de $+12,7 \%$.

\subsection{Des salaires variables, notamment selon le diplôme, la spécialité et le parcours d'études}

Le Tableau 3 présente les résultats de l'analyse réalisée en termes de salaires mensuels nets perçus par ceux qui occupent un emploi un an après l'obtention du diplôme. Il confirme, comme souligné dans d'autres études (Cipollone, Montanaro, Sestito, op. cit.) se référant également au cadre français (Dupray \& Barret, 2019), de fortes disparités selon le type d'études, où plus le niveau des études universitaires (premier/second cycle) est élevé, plus le salaire augmente : en effet, toutes choses égales par ailleurs, par rapport à un diplôme de premier cycle, l'obtention d'un master à cycle unique offre, en moyenne, un avantage salarial estimé à 260 euros nets mensuels ; être titulaire d'un master de deux ans d'études procure un avantage salarial de 55 euros. 
Tableau 3. Diplômés de premier et de second cycles de l'année 2017, interviewés un an après l'obtention du diplôme : modèle de régression linéaire pour l'évaluation du salaire mensuel net. Année d'enquête 2018

\begin{tabular}{|c|c|c|}
\hline & b & Erreur-type \\
\hline \multicolumn{3}{|l|}{ Genre (femmes $=0$ ) } \\
\hline Hommes & 84 & 3,84 \\
\hline Note de l'examen & 8 & 1,03 \\
\hline \multicolumn{3}{|l|}{ Type de cours (premier cycle $=0$ ) } \\
\hline Master de deux ans $(B A C+2)$ & 55 & 5,66 \\
\hline Master à cycle unique & 260 & 6,80 \\
\hline \multicolumn{3}{|l|}{ Domaine d'études (Socio-politique = 0 ) } \\
\hline Agriculture et études vétérinaires & -71 & 13,15 \\
\hline Architecture & -150 & 11,12 \\
\hline Chimie et pharmacologie & -6 & 11,84 \\
\hline Économie et statistique & 88 & 8,46 \\
\hline Éducation physique*** & -18 & 16,82 \\
\hline Géo-biologie*** & 9 & 12,26 \\
\hline Droit & -130 & 12,98 \\
\hline Ingénierie & 154 & 8,86 \\
\hline Enseignement ${ }^{* * *}$ & -13 & 11,86 \\
\hline Lettres & -49 & 10,76 \\
\hline Linguistique* & -25 & 10,03 \\
\hline Médecine et professions de la santé & 231 & 9,98 \\
\hline Psychologie & -41 & 14,59 \\
\hline Sciences & 137 & 12,29 \\
\hline \multicolumn{3}{|c|}{ Études à l'étranger (aucune expérience $=0$ ) } \\
\hline Erasmus - un autre programme de l'UE & 29 & 5,63 \\
\hline Autre expérience & 57 & 7,62 \\
\hline \multicolumn{3}{|c|}{ Travail pendant les études (aucun emploi $=0$ ) } \\
\hline Travailleur-étudiant & 98 & 11,68 \\
\hline Étudiant-travailleur & 11 & 3,66 \\
\hline \multicolumn{3}{|c|}{ Répartition géographique des emplois (Sud = 0 ) } \\
\hline Nord & 147 & 4,73 \\
\hline Centre & 76 & 5,58 \\
\hline Étranger & 436 & 8,89 \\
\hline \multicolumn{3}{|c|}{ Travail à temps plein / à temps partiel (temps partiel =0) } \\
\hline Temps plein & 385 & 4,71 \\
\hline
\end{tabular}




\begin{tabular}{|c|c|c|}
\hline \multicolumn{3}{|l|}{ Type de travail (non standard $=0$ ) } \\
\hline Indépendants* & -15 & 6,19 \\
\hline Durée indéterminée & 63 & 5,06 \\
\hline Contrats de formation & -64 & 5,42 \\
\hline Para-subordonné & -205 & 11,19 \\
\hline Autres autonomes & -270 & 8,66 \\
\hline Sans contrat & -397 & 10,44 \\
\hline \multicolumn{3}{|l|}{ Secteur d'activité (non marchand = 0) } \\
\hline Public & 144 & 10,27 \\
\hline Privé & 29 & 8,95 \\
\hline \multicolumn{3}{|c|}{$\begin{array}{l}\text { Domaine d'activité économique (services sociaux et personnels, récréatifs et } \\
\text { culturels }=0 \text { ) }\end{array}$} \\
\hline Agriculture & 81 & 18,94 \\
\hline Mécanique et mécanique de précision & 123 & 10,76 \\
\hline Bâtiment* & -25 & 11,83 \\
\hline Chimie/énergie & 118 & 11,02 \\
\hline Autre secteur manufacturier & 102 & 10,51 \\
\hline Commerce & 59 & 8,91 \\
\hline Crédit, assurance & 184 & 12,05 \\
\hline Transports, publicité, communications & 70 & 10,89 \\
\hline Conseils divers ${ }^{* * *}$ & 5 & 9,03 \\
\hline Informatique & 86 & 11,18 \\
\hline Autres services aux entreprises & 51 & 12,85 \\
\hline Administration publique, forces armées ${ }^{* * *}$ & 29 & 18,81 \\
\hline Éducation et recherche & -70 & 9,43 \\
\hline Santé & 112 & 8,49 \\
\hline \multicolumn{3}{|c|}{ Coordination formelle du travail effectué par d'autres personnes $($ non $=0$ ) } \\
\hline oui & 61 & 5,01 \\
\hline \multicolumn{3}{|c|}{ Efficacité du diplôme universitaire (peu efficace/pas du tout efficace $=0$ ) } \\
\hline Très efficace/efficace & 107 & 6,49 \\
\hline Assez efficace & 68 & 6,74 \\
\hline Constante & 225 & 29,13 \\
\hline
\end{tabular}

Pour chaque variable, nous avons considéré une modalité de référence (indiquée entre parenthèses près du nom de la variable) par rapport à laquelle sont calculés tous les coefficients $b$, et les erreurs-types, des autres modalités. Les coefficients représentent, par rapport à la modalité de référence, les augmentations en euro (diminution) lorsqu'ils sont supérieurs (inférieurs) à 0 . Par exemple, en ce qui concerne la variable du genre, on remarque que par rapport aux femmes, les hommes touchent 84 euros nets supplémentaires par mois.

Note: : R-carré $=0,469$ (R-carré ajusté $=0,468), \mathrm{N}=42114$.

* significativité à $5 \%(p<0,05)$ - *** Non significatif.

Là où non epressément indiqué, paramètres significatifs à $1 \%(p<0,01)$.

Source : AlmaLaurea (2019b). 
Un effet déterminant sur les écarts de salaire des diplômés est lié, toutes choses égales par ailleurs, au domaine d'études. Les diplômés des groupes médecine et professions de la santé, ingénierie, sciences et économie-statistique perçoivent, en moyenne, des salaires significativement supérieurs : par rapport aux diplômés du groupe socio-politique, le bonus salarial varie entre 231 et 88 euros nets par mois.

À l'inverse, les diplômés en architecture, droit, agriculture et études vétérinaires s'avèrent désavantagés du point de vue salarial : dans ce cas, la pénalité salariale, toujours par rapport aux diplômés du groupe socio-politique, oscille entre - 150 et - 71 euros nets par mois. Le fait qu'il s'agisse de parcours d'études où il est fréquent de démarrer des activités professionnelles libérales laisse supposer que la valorisation économique nécessite davantage de temps d'observation pour atteindre sa pleine expression.

Les différences traditionnelles de genre sont confirmées : toutes choses égales par ailleurs, les hommes touchent en moyenne, un an après le diplôme, 84 euros nets de plus par mois que les femmes. Des écarts territoriaux de salaires s'observent également. Ainsi, par rapport à ceux qui travaillent au Sud, ceux qui exercent au Nord perçoivent, en moyenne, 147 euros nets de plus par mois, tandis que ceux qui travaillent au Centre touchent 76 euros de plus. Mais c'est surtout par rapport aux diplômés qui travaillent à l'étranger que l'avantage salarial est le plus sensible (436 euros en plus). Bien sûr, il faudrait aussi tenir compte des disparités en termes de coût de la vie, en particulier par rapport à ceux qui se déplacent pour travailler à l'étranger, car cet élément a un impact sur les salaires, comme le soulignent de récentes études (Antonelli \& al., 2016 ; Chiesi \& Girotti, 2016).

Les expériences professionnelles, tout comme les études à l'étranger lors du parcours universitaire, impactent positivement les salaires mensuels nets, un an après l'obtention du diplôme. En particulier, toutes choses égales par ailleurs, les travailleurs-étudiants perçoivent 98 euros de plus que les diplômés ne possédant aucune expérience professionnelle. De même, ceux qui ont effectué une période d'études à l'étranger, reconnue dans leur cursus, touchent un salaire supérieur par rapport à ceux qui n'ont pas acquis cette expérience : par exemple, ceux qui ont étudié à l'étranger dans le cadre d'un programme de l'Union européenne peuvent espérer un gain salarial mensuel net de 29 euros.

Si l'on analyse à présent les caractéristiques de l'emploi occupé, outre l'effet important lié à la variable temps plein/temps partiel, il est intéressant d'observer les différences en termes contractuels : le modèle montre, ceteris paribus, des différences de salaires importantes. En effet, par rapport aux diplômés embauchés sous contrat non standard, ceux qui le sont sous contrat à durée indéterminée perçoivent 63 euros mensuels nets de plus. Les écarts salariaux sont particulièrement négatifs s'agissant d'activités qui ne sont réglementées par aucune forme contractuelle, d'activités de collaboration occasionnelle (" autres autonomes ", dans le tableau) et d'activités para-subordonnées : la perte salariale par rapport aux contrats non standards oscille entre - 397 et -205 euros nets par mois. De même, ceux qui sont embauchés sous contrat de formation, tout comme les indépendants, perçoivent un salaire inférieur à celui des travailleurs embauchés sous contrat non 
standard, mais dans ce cas, le préjudice salarial est moins marqué (respectivement, - 64 et - 15 euros). Les résultats de l'analyse confirment ceux d'autres études qui démontrent qu'en Italie, les contrats à durée déterminée ne sont pas compensés par des salaires plus élevés (ISTAT, 2018b). Le secteur et la branche de l'activité économique ont une incidence significative sur les salaires des diplômés. Toutes choses égales par ailleurs, par rapport au secteur non marchand, un emploi dans l'administration publique offre un avantage salarial de 144 euros, et de 29 euros dans le secteur privé. Sans doute que le secteur public, s'appuyant sur des mécanismes de recrutement par concours, garantit une reconnaissance formelle au diplôme possédé.

Enfin, l'efficacité du diplôme, mesurée à l'aune de la correspondance entre les études suivies et la profession exercée, exerce un effet positif sur le salaire des diplômés : bien qu’il puisse y avoir quelques risques d'endogénéité, le résultat semble intéressant car, toutes choses égales par ailleurs, ceux qui jugent leur diplôme très efficace ou efficace par rapport à l'emploi occupé perçoivent 107 euros de plus que ceux qui le jugent peu ou pas du tout efficace. L'efficacité du diplôme représente une mesure subjective de matching (adéquation) de l'emploi qui, comme l'ont démontré d'autres études (Ferrante, McGuinness, Sloane, 2010 ; Caroleo \& Pastore, 2013), est généralement positivement corrélée avec le salaire perçu.

\section{Conclusion}

La présente contribution visait à évaluer le capital humain et le rendement des études, à partir de l'analyse du contexte du marché du travail italien. Elle s'est donc centrée sur les débouchés des diplômés sur le marché du travail. L'évaluation des mécanismes à la base des débouchés et d'éventuelles conditions de chômage ou de sous-valorisation économique, est importante. Elle permet, en effet, de prendre en compte les implications associées aux "trigger events" (Di Prete, 2002), événements déclencheurs de mécanismes de stratification sociale qui conditionnent les trajectoires d'emploi des travailleurs, diplômés universitaires compris. Dans ce cadre, le fait d'être au chômage, déterminé par des périodes de crise économique prolongée, est clairement un exemple d'évènement pouvant impacter fortement les mécanismes de disparité (Gangl, op. cit.).

Les données d'AlmaLaurea plus récentes, se référanr à 2018, montrent des signes d'amélioration du marché du travail des diplômés, en Italie, après les années de crise, même si des difficultés, également structurelles, persistent, et que ces données enregistrent des disparités significatives selon le domaine d'études. Les débouchés sur le marché du travail des diplômés sont par ailleurs étroitement liés aux politiques et aux mesures législatives qui se sont succédé au cours des vingt dernières années : dans ce cadre, les politiques européennes de soutien à l'accès à l'emploi des jeunes, renforcées suite à la crise économique, n'ont pas constitué un nouveau cadre d'intervention, mais sont le résultat d'une logique incrémentielle (Lahusen, Schulz, Graziano, 2013). À la lecture des principaux indicateurs examinés, il est évident que ceux qui ont obtenu leur diplôme au cours des 
années les plus critiques de la crise et qui ont donc eu accès au marché du travail au cours de cette période, ont éprouvé de plus grandes difficultés. Dès lors, il semble que ces générations auront besoin de plus de temps pour surmonter ces difficultés. Cette hypothèse, confirmée par le fait que le capital humain se détériore s’il n’est pas opportunément valorisé, pointe le risque d'une déperdition potentielle de capital humain en cas de périodes de chômage prolongées, comme c'est le cas dans toutes les situations où un évènement déclencheur entraîne des répercussions tout au long de la vie active (Gangl, op. cit.).

Si le choix d'investir en capital humain s'envisage comme un choix individuel, les implications de ce choix sont aussi de nature sociale. Dans ce cadre, les marges d'intervention sont plutôt vastes. Les données d'AlmaLaurea révèlent un écart de rendement lié au niveau des études universitaires : en effet, les diplômés de second cycle peuvent espérer une meilleure valorisation économique. Selon certaines études (Villosio, 2010), une des causes de ce résultat réside dans les contenus des enseignements de premier cycle et leur inadaptation aux exigences du marché du travail. Cette hypothèse suppose ainsi que les employeurs soient en mesure d'apprécier la différence de préparation entre des diplômés de premier et de second cycles. Les caractéristiques structurelles du marché du travail italien suscitent des interrogations légitimes à cet égard, puisqu'elles révèlent les limites d'un système entrepreneurial, caractérisé par la primauté des petites entreprises à gestion familiale (ISTAT, 2018a ; Bugamelli \& al., op. cit.) qui, globalement, sont révélatrices d'une moindre propension à valoriser la connaissance (AlmaLaurea, 2015). Mais, en faisant abstraction de ces évaluations, la promotion de certains enseignements de premier cycle professionnel, mise en œuvre de façon expérimentale à partir de l'année académique 2018/19 afin de former des profils professionnels hautement spécialisées (Fondazione CRUI, 2019), constitue une réponse concrète du système universitaire italien à ce problème.

Parallèlement, il serait souhaitable d'enrichir également davantage les enseignements des masters universitaires, cursus à haut contenu professionnel auxquels on accède avec un diplôme universitaire (à ne pas confondre avec les diplômes dénommés " master " des systèmes universitaires étrangers). L'avantage de ces parcours de formation est la flexibilité : s'agissant, typiquement, d'enseignements d'une durée annuelle, leur conception et leur adaptation sont plus souples, aspect non négligeable pour la conception de parcours d'études devant répondre aux impératifs du marché du travail.

S'agissant du troisième cycle du système universitaire italien, des doctorats industriels ont ainsi vu le jour. Ce parcours sont réalisés en partenariat avec des entreprises afin de former des docteurs spécialité " recherche ", susceptibles de mettre leurs compétences au service des entreprises : il s'agit d'un nouvel apport (quoiqu'encore modeste sur le plan numérique) et d'un exemple vertueux d'interaction entre les universités et les entreprises, qui s'avère décisif pour promouvoir l'innovation, la recherche et le développement.

De même, il faudrait miser sur des mesures comme les avantages fiscaux et les primes à l'embauche, afin de promouvoir le capital humain le plus formé et le mieux préparé. Les contrats d'apprentissage de haute formation et recherche (D. L. $\mathrm{n}^{\circ}$ 81/2015) s'inscrivent 
dans ce sillon (ils font partie des contrats de formation, dans les enquêtes AlmaLaurea). Ce sont des formes de travail salarié destinées à la formation et à l'emploi des jeunes âgés de 18 à 29 ans, et qui prévoient à la fois l'embauche dans une entreprise et la possibilité d'obtenir un diplôme d'études universitaires ou d'effectuer des activités de recherche. Toutes ces options permettent aux entreprises d'intégrer des profils professionnels de niveaux moyens-élevés dans leurs effectifs, pour apporter de l'innovation et accroître la productivité.

La question du niveau de l'enseignement supérieur de la population italienne est loin d'être résolue. Il faut investir, selon une logique de filière, dans l'école, dans l'université, dans la recherche et l'innovation, et plus largement, en capital fixe (physique et immatériel), essentiel pour promouvoir le capital humain le plus qualifié (AlmaLaurea, 2014). La dotation, quantitative et qualitative, en capital humain a contribué à définir le modèle de spécialisation de la production des entreprises italiennes, et en a malheureusement limité la valorisation de l'investissement, en décourageant son accumulation (Cipollone, Montanaro, Sestito, op. cit.) et en déclenchant un véritable cercle vicieux (Visco, 2009). Comme le soulignent certaines études (Franco, 2012), un des motifs expliquant la faiblesse de la croissance talienne, au cours des quinze dernières années, est justement l'incapacité à valoriser les opportunités offertes par les Technologies de l'Information et de la Communication. Les propositions ci-après pourraient contribuer à revigorer le système italien

S'agissant des disparités territoriales, les données d'AlmaLaurea confirment que l'Italie avance sur deux rails parallèles, mais à des vitesses différentes. Plus généralement, le Nord du pays est doté d'une structure productive et d'une économie de même niveau que celle des principaux partenaires européens, tandis que le Sud éprouve de graves difficultés à soutenir les rythmes de croissance nécessaires (ISTAT, 2019d ; Svimez, 2018). La crise a par ailleurs aggravé l'écart territorial (Stella \& Rizzo, 2015 ; Viesti, 2016) avec le Sud, qui connaît un profond changement démographique, en lien également avec les migrations (Svimez, op. cit.). Ces difficultés, qui plongent toutefois leurs racines dans des périodes historiques bien plus lointaines, se sont accrues ces dernières années. Elles concernent différents domaines sociaux, qu'il s'agisse de l'éducation et de la formation ou du système économique et productif. Tout ceci révèle l'urgence d'une réflexion sur les stratégies à mettre en œuvre pour relancer l'ensemble du système italien, notamment s'agissant des régions les plus fragiles. Le recours à l'entreprenariat et à l'auto-entreprenariat pourrait être une des réponses possibles aux difficultés d'un contexte, comme celui du Sud, apparemment encore inerte aujourd'hui face aux défis du changement. Pour autant, des signes positifs existent (Cannari, Magnani, Pellegrini, 2009). Il sont aussi confirmés par les enquêtes d'AlmaLaurea : au Sud, les activités de type autonome et entrepreneuriales sont relativement plus fréquentes. Il s'agirait de promouvoir la culture de l'entreprenariat 
parmi les étudiants universitaires, à travers l'activation de spin-off académiques ${ }^{9}$, en encourageant les start-ups à une contribution technologique significative, ou plus simplement par la promotion d'activités interdisciplinaires sur ces thèmes. Il faut dire que l'intérêt du système universitaire est croissant (Fondazione CRUI, 2016) : il convient donc d'encourager, in primis, les jeunes, pour investir et attirer également des capitaux dans le Sud. Des initiatives législatives allant dans ce sens ont déjà montré leur efficacité, comme en attestent les résultats obtenus pendant la première moitié des années 90 en termes d'investissements, d'immobilisations et d'emplois (Pellegrini \& Carlucci, 2003). Mais ces propositions ne suffisent pas à elles seules. "Il faut mettre en cuvre des politiques pour la croissance économique, des mesures pour la formation et la recherche, un réseau efficace de transport dans toutes les régions, mesures aptes à créer les conditions d'un contexte favorable à l'activité des entreprises pouvant les conduire à augmenter partout leur effort d'investissement, notamment là où, comme au Sud, il existe des opportunités non utilisées" (Viesti, 2003).

\section{Bibliographie}

Ardilly P. (2006), Les techniques de sondage, Paris ? Éditions Technip.

AlmaLaurea (2019a), XXI Indagine. Profilo dei Laureati 2018. Rapporto 2019, Bologna.

AlmaLaurea (2019b), XXI Indagine. Condizione occupazionale dei Laureati. Rapporto 2019, Bologna.

AlmaLaurea (2015), Condizione occupazionale dei Laureati. XVII Indagine 2014, Bologna.

AlmaLaurea (2014), Condizione occupazionale dei Laureati. XVI Indagine 2013, Bologna.

Antonelli G., Binassi S., Guidetti G., Pedrini G. (2016), “Assessing selection patterns and wage differential of high-skilled migrants. Evidence from the AlmaLaurea dataset on Italian graduates working abroad”, AlmaLaurea Working Papers, n ${ }^{\circ} 76$, Bologna.

ANVUR (2018), Rapporto sullo stato del sistema universitario e della ricerca 2018, Roma.

Bailey K. D. (2006), Metodi della ricerca sociale. I. I principi fondamentali, Bologna, Il Mulino.

Banca d'Italia (2015), Relazione Annuale sul 2014, Roma.

Becker G. S. (1964), Human capital theory, New York, Columbia.

9. (Ou "essaimage universitaire")... "nous retiendrons la définition de Smilor et al. (1990) qui parlent d'ailleurs de Research Based spin-offs (RBSO) plutôt que de spin-off. Pour eux, "une RBSO correspond à la création d'une entreprise (1) par un membre du personnel de l'université ou par un étudiant qui quitte l'université pour créer une entreprise, ou qui démarre une entreprise alors qu'il est encore attaché à l'université ; etlou (2) sur la base d'une technologie développée au sein de l'université" : Consulté sur : https://www.cairn.info/revue-de-l-entrepreneuriat-2015-1-page-41.htm? contenu=resume 
Bender S., Dustmann C., Margolis D., Meghir C. (2002), "Worker Displacement in France and Germany", in Losing Work, Moving On: International Perspectives on Worker Displacement.

Bernardi F. \& Ballarino G. (a cura di) (2016), Education, Occupation and Social Origin: A Comparative Analysis of the Transmission of Socio-Economic Inequalities, Edward Elgar Publishing.

Biagi M. (a cura di) (1997), Mercati e rapporti di lavoro: commentario alla Legge 24 giugno 1997, n ${ }^{\circ} 196$, Milano, Giuffrè.

Biggeri L. (2006), Indagine conoscitiva sulle cause e le dimensioni del precariato nel mondo del lavoro, Audizione del Presidente dell'Istat alla Camera dei Deputati, XI Commissione 'Lavoro pubblico e privato'.

Bugamelli M., Cannari L., Lotti F., Magri S. (2012), Il gap innovativo del sistema produttivo italiano : radici e possibili rimedi, Roma, Banca d'Italia.

Camillo F., Conti V., Ghiselli S. (2011), "Integration of different data collection techniques using the propensity score”, AlmaLaurea Working Papers, $\mathrm{n}{ }^{\circ} 4$, Bologna.

Cannari L., Magnani M., Pellegrini G. (2009), Quali politiche per il Sud? Il ruolo delle politiche nazionali e regionali nell'ultimo decennio, Roma, Banca d'Italia.

Cappelli P. H. (2015), "Skill gaps, skill shortages, and skill mismatches: Evidence and arguments for the United States", in ILR Review, n ${ }^{\circ} 68(2)$.

Caroleo F. E., Pastore F. (2013), "L'overeducation in Italia : le determinanti e gli effetti salariali nei dati AlmaLaurea", in Scuola democratica, n ${ }^{\circ} 4(2)$.

Chiesi A. M., Girotti C. (2016), "Retribuzioni dei laureati e mercato del lavoro in tempi di crisi”, in Quaderni di sociologia, $\mathrm{n}^{\circ} 72$, Torino, Rosenberg \& Sellier.

Cipollone P., Montanaro P. \& Sestito P. (2012), Il capitale umano per la crescita economica: possibili percorsi di miglioramento del sistema d'istruzione in Itali, Roma, Banca d'Italia.

Cipollone P., Sestito P. (2010), Il capitale umano, Il Mulino, Bologna.

Consiglio dell'Unione europea (2018), Le raccomandazioni e i pareri sulle politiche economiche, occupazionali e di bilancio degli Stati membri per il 2018, Bruxelles.

Deming W. E., Stephan F. F. (1940), “On a least square adjustment of a sampled frequency table when the expected marginal totals are known", in The Annals of Mathematical Statistics, $\mathrm{n}^{\circ} 11$, pp. 427-444.

Di Prete T. A. (2002), "Life Course Risks, Mobility Regimes, and Mobility Consequences: A Comparison of Sweden, Germany, and the United States", American Journal of Sociology, $\mathrm{n}^{\circ} 108$. 
Dupray A., Barret C. (2019), "Que gagne-t-on à se former ? Zoom sur 20 ans d'évolution des salaires en début de vie active ", Céreq Bref, n ${ }^{\circ} 372$.

Eurostat (2019a), Labour Force Survey. Employment and activity by sex and age - annual data, http://ec.europa.eu/eurostat/web/lfs/data/database.

Eurostat (2019b), Tertiary educational attainment by sex, age group 30-34, http://ec.europa. eu/eurostat $/ \mathrm{tgm} / \mathrm{table}$. do?tab=table\&init=1 \&language $=$ en $\&$ pcode $=t 2020 \_41 \& \mathrm{pl}$ ugin $=1$.

Eurostat (2019c), Gross domestic expenditure on R\&D, http://ec.europa.eu/eurostat/tgm/ table.do?tab=table\&init=1\&language $=$ en $\&$ pcode=t2020_20\&plugin $=1$.

Ferrante F., McGuinness S., Sloane P. J. (2010), “Esiste 'overeducation'? Un'analisi comparata", in AlmaLaurea, XII Rapporto sulla condizione occupazionale dei laureati. Investimenti in capitale umano nel futuro di Italia ed Europa, Bologna, Il Mulino.

Fondazione CRUI-Osservatorio Università-Imprese (2019), OU-I 2018, Roma.

Fondazione CRUI-Osservatorio Università-Imprese (2016), OU-I 2015, Roma.

Franco D. (2012), Indagine conoscitiva sulle caratteristiche e sullo sviluppo del sistema industriale, delle imprese pubbliche e del settore energetico, Roma.

Galeazzi S., Ghiselli S. (2016), "Ruolo della famiglia nelle scelte formative e nelle esperienze maturate durante il percorso di studio", in Nuti S., Ghio A. (a cura di), Obiettivo mobilità sociale. Sostenere il merito per creare valore nel sistema Paese, Bologna, Il Mulino.

Gangl M. (2006), "Scar effects of unemployment: An assessment of institutional complementarities", American Sociological Review n ${ }^{071}$, pp. 986-1013.

Gregory M., Jukes R. (2001), “Unemployment and Subsequent Earnings: Estimating Scarring among British Men 1984-1994”, Economic Journal, n²111, pp. F607-F625.

Hanushek E. A., Woessmann L. (2009), "Do Better Schools Lead to More Growth? Cognitive Skills, Economic Outcomes, and Causation”, NBER Working Paper n ${ }^{\circ} 14633$.

ISTAT (2019a), Rapporto sulla competitività dei sistemi produttivi. Edizione 2019, Roma.

ISTAT (2019b), Il mercato del lavoro 2018. Verso una lettura integrata, Roma.

ISTAT (2019c), Noi Italia. 100 statistiche per capire il Paese in cui viviamo, Roma.

ISTAT (2019d), Rapporto annuale 2019. La situazione del Paese, Roma.

ISTAT (2019e), Rilevazione sulle forze di lavoro, http://dati.istat.it/Index. aspx? DataSetCode=DCCV_TAXDISOCCU1.

ISTAT (2019f), FOI(nt) - Indici nazionali dei prezzi al consumo per le famiglie di operai e impiegati. Coefficienti per tradurre valori monetari dei periodi sottoindicati in valori del 2018, www.istat.it/it/archivio/30440; 
ISTAT (2018a), Rapporto sulla conoscenza 2018. Economia e società, Roma.

ISTAT (2018b), I differenziali retributivi nel settore privato. Anni 2014-2016, Roma.

Johnes G. (1993), The economics of education, Macmillan International Higher Education.

Lahusen C., Schulz N., Graziano P. R. (2013), "Promoting social Europe? The development of European youth unemployment policies", International journal of social welfare $n^{\circ} 22.3$.

Layte R., Levin H., Hendrickx J., Bison I. (2000), "Unemployment and Cumulative Disadvantage in the Labour Market", in Welfare Regimes and the Experience of Unemployment in Europe, Oxford.

Lopez A., Sulzer E. (2016), "Apprentices and the training-to-work transition: an unqualified advantage?”, Training \& Employment, n 123.

Luzzatto G., Mangano S. \& Moscati R. (2011), "Bachelor degree owners' employment in Italy and in other European countries”, AlmaLaurea Working Papers, n ${ }^{\circ} 43$.

Massagli E. (2018), Piccolo compendio delle riforme del lavoro dal 1997 al 2018 : dalla 'qualită alla 'dignită', Bergamo, Adapt University Press.

McGuinness S., Sloane P. J. (2011), "Labour market mismatch among UK graduates: An analysis using REFLEX data", in Economics of Education Review, n 30(1).

McGuinness S., Whelan A., Bergin A. (2016) "Is there a role for higher education institutions in improving the quality of first employment?", in The BE Journal of Economic Analysis \& Policy $\mathrm{n}^{\circ} 16$.

OECD (2018), Education at a Glance 2018:OECD Indicators, Paris, OECD Publishing.

Oreopoulos P., von Wachter T., Heisz A. (2006), “The Short- and Long-Term Career Effects of Graduating in a Recession: Hysteresis and Heterogeneity in the Market for College Graduates”, NBER WP n 12859.

Pellegrini G., Carlucci C. (2003), “Gli effetti della legge 488/92 : una valutazione dell'impatto occupazionale sulle imprese agevolate", in Rivista Italiana degli Economisti, n ${ }^{\circ} 2$.

Praussello F., Marenco M. (1996), Economia dell'istruzione e del capitale umano, Bari, Laterza.

Regini M. (2014), La riforma universitaria nel quadro dei sistemi di governance europei, $\mathrm{n}^{\circ} 8$, Firenze, Firenze University Press.

Stella G. A., Rizzo S. (2015), Se muore il Sud, Milano, Feltrinelli.

Svimez (2018), Rapporto Svimez 2018. L'economia e la società del Mezzogiorno, Bologna, Il Mulino. 
Tridico P. (2014), "Riforme del mercato del lavoro, occupazione e produttività : un confronto tra l'Italia e l'Europa”, in Sindacalismo, n 28.

UniversItaly, www.universitaly.it.

USTAT, http://ustat.miur.it/.

Vaira M. (2011), La costruzione della riforma universitaria e dell'autonomia didattica: idee, norme, pratiche, attori, Milano, LED Edizioni Universitarie.

Viesti G. (2016), Università in declino, Roma, Donzelli.

Viesti G. (2003), Abolire il Mezzogiorno, Bari, Laterza.

Villosio C. (2010), "I nuovi laureati al giudizio dei direttori del personale", FGA Working Paper n ${ }^{\circ} 35$.

Visco I. (2009), Investire in conoscenza, Bologna, Il Mulino. 